\title{
Residuated Kleene Algebras
}

\author{
Hajnal Andréka ${ }^{1}$, Szabolcs Mikulás ${ }^{2}$, and István Németi ${ }^{1}$ \\ 1 Alfréd Rényi Institute of Mathematics \\ Hungarian Academy of Sciences \\ 13-15 Reáltanoda u., 1053 Budapest, Hungary \\ andreka@renyi.hu and nemeti@renyi.hu * \\ 2 Department of Computer Science and Information Systems \\ Birkbeck, University of London \\ Malet Street, London WC1E 7HX, UK \\ szabolcs@dcs.bbk.ac.uk
}

\begin{abstract}
We show that there is no finitely axiomatizable class of algebras that would serve as an analogue to Kozen's class of Kleene algebras if we include the residuals of composition in the similarity type of relation algebras.
\end{abstract}

\section{Introduction}

One of the standard interpretations of Kleene algebras is by families of binary relations: relational Kleene algebras, RKA. It is well known that the equational theory of RKA is not finitely axiomatizable; see [14] or [4, Theorem 9] in English. But Kozen [9] proved that there are intuitive quasi-equations (i.e., equational implications) valid in RKA which together with finitely many equations do axiomatize the equational theory of RKA. The so obtained quasi-variety is Kozen's class of Kleene algebras, KA. Thus there is a finitely axiomatized quasi-variety $\mathrm{KA} \supseteq$ RKA generating the same variety as RKA, i.e., using the terminology of Definition 2.3,

KA provides a strong, finite quasi-axiomatization of RKA.

Pratt [13] observed that including the residuals of composition into the similarity type of Kleene algebras has the advantage that the resulting class is a finitely axiomatizable variety; with the use of the residuals the quasi-equations in the axiomatization of KA can be expressed as equations. Following Pratt we will call the class of Kleene algebras equipped with the residuals of composition action algebras, $\mathrm{AA}$, and we will call the subclass of action algebras that can be interpreted over families of binary relations relational action algebras, RAA. Thus, RAA consists of members of RKA equipped with the residuals.

In this paper we prove that the price of including the residuals into the similarity type, and so turning KA into a variety $\mathrm{AA}$, is not only that the equational theory of RAA is not finitely axiomatizable, but there is no strong, finite

* Research supported by the Hungarian National Foundation for Scientific Research grant No. T81188. 
quasi-axiomatization for RAA; see Theorem 3.2. Thus, the "trick" in defining KA cannot be done again for RAA.

Kozen [10] expands the similarity type of action algebras by meet. The resulting class, action lattices, AL, is again a finitely axiomatizable variety. We define the class of relational action lattices, RAL, as that subclass of AL whose elements can be represented on binary relations. The equational theory of RAL turns out to be nonfinitely axiomatizable; see [7]. Here we give an alternative, simpler proof of this fact, and show that strong, finite quasi-axiomatization is impossible in this case, too; see Theorem 4.1.

The rest of the paper is organized as follows. In the next section we give precise definitions of the classes of algebras to be investigated and a formal definition of finite quasi-axiomatizability. Then we turn our attention to action algebras in Section 3. In Section 4 we look at action lattices. We conclude with some open problems.

\section{Basics}

Given a similarity type $\Lambda$, we denote the class of relational $\Lambda$-algebras by $\mathrm{R}(\Lambda)$ : the class of those $\Lambda$-algebras that are isomorphic to algebras of binary relations where the elements of $\Lambda$ are interpreted as "natural" operations on binary relations. Using the terminology of the relation algebra literature, we will sometimes refer to elements of $\mathrm{R}(\Lambda)$ as representable algebras. Below we give precise definitions of $\mathrm{R}(\Lambda)$ for particular choices of $\Lambda$.

Definition 2.1 (Relational Kleene algebras). The class of relational Kleene algebras is

$$
\mathrm{RKA}=\mathrm{R}\left(+, ;,{ }^{*}, 0,1^{\prime}\right),
$$

i.e., the class of subalgebras of algebras of the form $\left(\wp(W),+, ;,{ }^{*}, 0,1^{\prime}\right)$ where $W$ is an equivalence relation, + is set union, ; is relation composition

$$
x ; y=\{(u, v) \in W:(u, w) \in x \text { and }(w, v) \in y \text { for some } w\},
$$

* is reflexive-transitive closure, 0 is the emptyset, and $1^{\prime}$ is the identity relation restricted to $W$

$$
1^{\prime}=\{(u, v) \in W: u=v\} .
$$

We recall the interpretation of the residuals $\backslash$ and / of composition in relation algebras:

$$
\begin{aligned}
& x \backslash y=\{(u, v) \in W: \forall w((w, u) \in x \text { implies }(w, v) \in y)\} \\
& x / y=\{(u, v) \in W: \forall w((v, w) \in y \text { implies }(u, w) \in x)\} .
\end{aligned}
$$

Next we define expansions of relational Kleene algebras with residuals and meet; see $[13,10,8]$ for similar expansions. 
Definition 2.2 (Relational residuated Kleene algebras). The class of relational action algebras is defined as

$$
\mathrm{RAA}=\mathrm{R}\left(+, ;{ }^{*}, /, \backslash, 0,1^{\prime}\right)
$$

while the class of relational action lattices is defined as

$$
\mathrm{RAL}=\mathrm{R}\left(\cdot,+, ;{ }^{*}, /, \backslash, 0,1^{\prime}\right)
$$

where is interpreted as intersection.

We note that we would get an equivalent definition if we require that $W$ is a Cartesian square $U \times U$ in the above definitions. We chose $W$ be an equivalence relation, since we will sometimes include the top element 1 into $\Lambda$, and requiring that 1 is interpreted as a Cartesian square would result in classes that are not closed under products. Other additional operations we will consider in this paper are complement - and converse ${ }^{\smile}$. See $[5,6]$ for Kleene algebras expanded with converse.

It may be useful to introduce some terminology to describe when the equational theory of a class of algebras has a finite quasi-equational axiomatization.

Definition 2.3. Given a class $\mathrm{K}$ of algebras, we say that (the equational theory of) $\mathrm{K}$ is finitely quasi-axiomatized if there is a quasi-variety $\mathrm{Q}$ such that

- $\mathrm{Q}$ and $\mathrm{K}$ generate the same variety, i.e., their equational theories coincide: $\mathrm{Eq}(\mathrm{Q})=\mathrm{Eq}(\mathrm{K})$,

$-\mathrm{Q}$ is finitely axiomatizable.

If, in addition,

$-\mathrm{K} \subseteq \mathrm{Q}$, i.e., the axioms of $\mathrm{Q}$ are valid in $\mathrm{K}$,

then we say that the finite quasi-axiomatization is strong.

\section{Action Algebras}

In this section we show that relational action algebras do not have a strong, finite quasi-axiomatization; see Theorem 3.2.

It is well known that the classes RAA and RAL are not axiomatizable by first-order logic formulas, because the presence of reflexive-transitive closure in their signatures causes them to be not closed under ultraproducts. The following theorem cited from [2, Theorem 5.1] implies that even their quasi-equational theories are not finitely axiomatizable.

Theorem 3.1. Let $\{+, ;\} \subseteq \Lambda \subseteq\left\{+, ;,{ }^{*}, /, \backslash,{ }^{\smile}, 0,1^{\prime}, 1\right\}$. Neither $\mathrm{R}(\Lambda)$ nor the quasi-equational theory of $\mathrm{R}(\Lambda)$ is finitely axiomatizable. 
Now, the equational theory of $\mathrm{R}(+, ;)$ is different, it is finitely axiomatizable (by equations), and moreover the equational theories of $\mathrm{R}(\Lambda)$ where the residuals and transitive closure are not included in $\Lambda$ tend to be finitely axiomatizable. For a complete description of the cases see [2]. Our next theorem shows that this situation changes radically if we include the residuals into the similarity types.

Theorem 3.2. Let $\{+, ;, /, \backslash\} \subseteq \Lambda \subseteq\left\{+, ;{ }^{*}, /, \backslash,{ }^{\smile}, 0,1^{\prime}, 1\right\}$. The equational theory of $\mathrm{R}(\Lambda)$ is not finitely axiomatizable.

Moreover, there is no strong, finite quasi-axiomatization of $\mathrm{R}(\Lambda)$. In fact, there is no first-order logic formula valid in $\mathrm{R}\left(+, ;,{ }^{*}, /, \backslash,{ }^{\smile}, 0,1^{\prime}, 1\right)$ which implies all the equations valid in $\mathrm{R}(+, ;, /, \backslash)$.

Proof. We recall, for every natural number $n$, the algebra

$$
\mathfrak{A}_{n}=\left(A_{n},+, ;,^{*}, /, \backslash,{ }^{\smile}, 0,1^{\prime}, 1\right)
$$

from [2, Theorem 5.1].

We define

$$
G_{n}=\left\{a, a_{1}^{\prime}, a_{1}^{\prime \prime}, \ldots, a_{n}^{\prime}, a_{n}^{\prime \prime}, b, b_{1}^{\prime}, b_{1}^{\prime \prime}, \ldots, b_{n}^{\prime}, b_{n}^{\prime \prime}, o, 1^{\prime}, 0\right\} .
$$

Let $\left(A_{n},+\right)$ be the free upper semilattice generated freely by $G_{n}$ under the defining relations:

$$
\left\{a \leq a_{i}^{\prime}+a_{i}^{\prime \prime}, b \leq b_{i}^{\prime}+b_{i}^{\prime \prime}, 0+x=x: 1 \leq i \leq n, x \in G_{n}\right\} .
$$

Let $S$ denote the following set of two-element subsets of $A_{n}$ :

$$
S=\left\{\left\{a, b_{1}^{\prime}\right\}\right\} \cup\left\{\left\{a_{i}^{\prime}, b_{i}^{\prime \prime}\right\}: 1 \leq i \leq n\right\} \cup\left\{\left\{a_{i}^{\prime \prime}, b_{i+1}^{\prime}\right\}: 1 \leq i<n\right\} \cup\left\{\left\{a_{n}^{\prime \prime}, b\right\}\right\} .
$$

Next we define the rest of the operations on $A_{n}$ as follows:

$$
\begin{aligned}
& 0=\emptyset \quad 1=\sum G_{n} \quad x^{\smile=x} \\
& 0 ; x=0=x ; 0 \quad 1^{\prime} ; x=x=x ; 1^{\prime} \\
& \text { if } x, y \notin\left\{0,1^{\prime}\right\}, \text { then } x ; y= \begin{cases}o & \text { if }\{x, y\} \in S \\
1 & \text { otherwise. }\end{cases}
\end{aligned}
$$

Kleene star * is defined as follows. We have $0 ; 0=0,1^{\prime} ; 1^{\prime}=1^{\prime}$ and $x ; x=1$ for every $x \in A_{n} \backslash\left\{0,1^{\prime}\right\}$. Hence we define, in $\mathfrak{A}_{n}, 0^{*}=1^{\prime}, 1^{\prime *}=1^{\prime}$ and $x^{*}=1$ for every $x \in A_{n} \backslash\left\{0,1^{\prime}\right\}$.

We define the residual $\backslash$ in the algebras $\mathfrak{A}_{n}$ so that $x \backslash y$ is the largest element $z$ such that $x ; z \leq y$. Then the algebras $\mathfrak{A}_{n}$ are in fact closed under the operation $\backslash$ (since they are finite). Indeed, the extension of $x \backslash y$ is determined by

$$
z \leq x \backslash y \text { iff } x ; z \leq y \text {. }
$$

Note that this defines / as well, since / and $\backslash$ coincide in symmetric algebras (where $x ; y=y ; x$ is valid).

It is shown in [2] that 
1. the $\{+, ;\}$-reduct of $\mathfrak{A}_{n}$ is not representable,

2. any nontrivial ultraproduct over $\omega, \mathfrak{A}$, of the $\mathfrak{A}_{n}$ 's is representable.

Item 1 above was shown by constructing a quasi-equation $q_{n}$ for every $n$ as

$$
\begin{aligned}
& \bigwedge_{i=1}^{n}\left(x \leq x_{i}^{\prime}+x_{i}^{\prime \prime} \wedge y \leq y_{i}^{\prime}+y_{i}^{\prime \prime}\right) \rightarrow \\
& x ; y \leq x ; y_{1}^{\prime}+\sum_{i=1}^{n-1}\left(x_{i}^{\prime} ; y_{i}^{\prime \prime}+x_{i}^{\prime \prime} ; y_{i+1}^{\prime}\right)+x_{n}^{\prime} ; y_{n}^{\prime \prime}+x_{n}^{\prime \prime} ; y .
\end{aligned}
$$

By an induction on $n$ one can show that $q_{n}$ is valid in representable algebras. On the other hand, the evaluation $\epsilon$ given by

$$
\epsilon(x)=a \quad \epsilon\left(x_{i}^{\prime}\right)=a_{i}^{\prime} \quad \epsilon\left(x_{i}^{\prime \prime}\right)=a_{i}^{\prime \prime} \quad \epsilon(y)=b \quad \epsilon\left(y_{i}^{\prime}\right)=b_{i}^{\prime} \quad \epsilon\left(y_{i}^{\prime \prime}\right)=b_{i}^{\prime \prime}
$$

falsifies $q_{n}$ in $\mathfrak{A}_{n}$ (since $a ; b=1$ and each term on the right of $\leq$ in the consequent evaluates to $o$ ).

Here we modify $q_{n}$ to equation $e_{n}$ with the same properties, but we have to pay the price of including the residuals into the language. Then it follows that the $\{+, ;, /, \backslash\}$-reduct of $\mathfrak{A}_{n}$ is not in the variety generated by the representable algebras.

Below we will use the following abbreviations $x_{i}:=x_{i}^{\prime}+x_{i}^{\prime \prime}$ and $y_{i}:=y_{i}^{\prime}+y_{i}^{\prime \prime}$ for $1 \leq i \leq n$. We define, inductively,

$$
\kappa_{1}=x \backslash x_{1} \quad \kappa_{i+1}=\left(x ; \kappa_{1} ; \ldots ; \kappa_{i}\right) \backslash x_{i+1}
$$

and

$$
\lambda_{1}=y_{n} / y \quad \lambda_{i+1}=y_{n-i+1} /\left(\lambda_{i} ; \ldots ; \lambda_{1} ; y\right) .
$$

Let $\tau_{n}$ be the term

$$
x ; \kappa_{1} ; \ldots ; \kappa_{n} ; \lambda_{n} ; \ldots ; \lambda_{1} ; y \text {. }
$$

We define $\sigma_{n}$ as

$$
\begin{aligned}
& x ; \kappa_{1} ; \ldots ; \kappa_{n} ; y_{1}^{\prime}+x_{1}^{\prime} ; \kappa_{2} ; \ldots ; \kappa_{n} ; y_{1}^{\prime \prime}+x_{1}^{\prime \prime} ; \kappa_{2} ; \ldots ; \kappa_{n} ; \lambda_{n} ; y_{2}^{\prime}+\ldots+ \\
& x_{n-1}^{\prime \prime} ; \kappa_{n} ; \lambda_{n} ; \ldots ; \lambda_{2} ; y_{n}^{\prime}+x_{n}^{\prime} ; \lambda_{n} ; \ldots ; \lambda_{2} ; y_{n}^{\prime \prime}+x_{n}^{\prime \prime} ; \lambda_{n} ; \ldots ; \lambda_{1} ; y .
\end{aligned}
$$

Finally, $e_{n}$ is defined as $\tau_{n} \leq \sigma_{n}$.

It is not difficult to show that $e_{n}$ is valid in representable algebras. As an example we show the case $n=2$. See Figure 1 .

Equation $e_{2}$ has the form $\tau_{2} \leq \sigma_{2}$ where

$$
\tau_{2}=x ; x \backslash x_{1} ;\left(x ; x \backslash x_{1}\right) \backslash x_{2} ; y_{1} /\left(y_{2} / y ; y\right) ; y_{2} / y ; y
$$




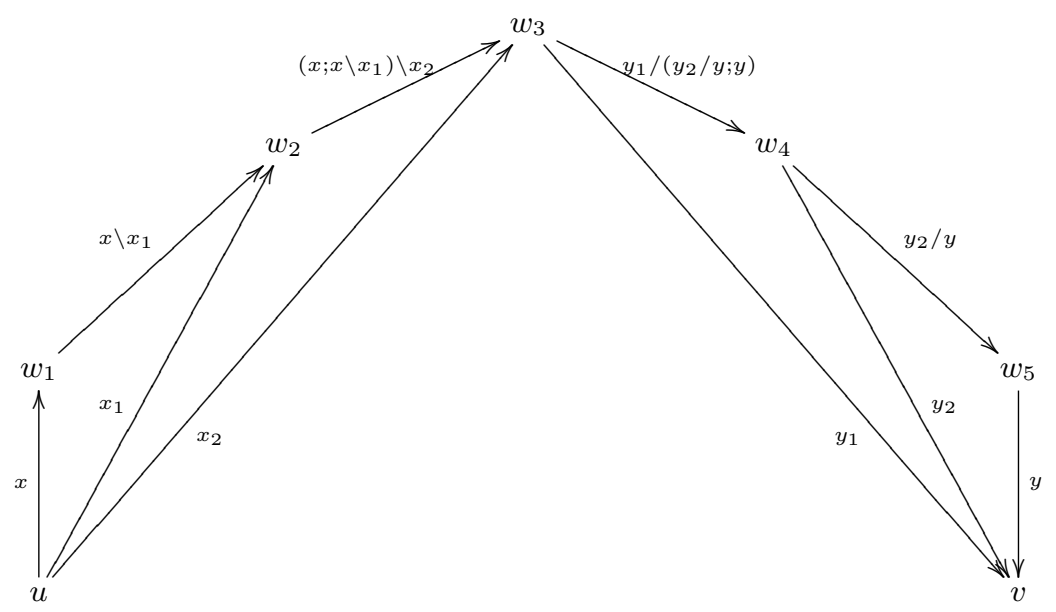

Fig. 1. Validity of $e_{2}$

and

$$
\begin{aligned}
\sigma_{2}= & x ; x \backslash x_{1} ;\left(x ; x \backslash x_{1}\right) \backslash x_{2} ; y_{1}^{\prime}+ \\
& x_{1}^{\prime} ;\left(x ; x \backslash x_{1}\right) \backslash x_{2} ; y_{1}^{\prime \prime}+ \\
& x_{1}^{\prime \prime} ;\left(x ; x \backslash x_{1}\right) \backslash x_{2} ; y_{1} /\left(y_{2} / y ; y\right) ; y_{2}^{\prime}+ \\
& x_{2}^{\prime} ; y_{1} /\left(y_{2} / y ; y\right) ; y_{2}^{\prime \prime}+ \\
& x_{2}^{\prime \prime} ; y_{1} /\left(y_{2} / y ; y\right) ; y_{2} / y ; y .
\end{aligned}
$$

Let $\mathfrak{A}$ be a representable algebra and assume that $(u, v) \in \tau_{2}$ in $\mathfrak{A}$ under some valuation (for the sake of simplicity we denote the value of a term as the term itself). Then there are $w_{1}, w_{2}, \ldots, w_{5}$ such that $\left(u, w_{1}\right) \in x,\left(w_{1}, w_{2}\right) \in x \backslash x_{1}$, $\left(w_{2}, w_{3}\right) \in\left(x ; x \backslash x_{1}\right) \backslash x_{2},\left(w_{3}, w_{4}\right) \in y_{1} /\left(y_{2} / y ; y\right),\left(w_{4}, w_{5}\right) \in y_{2} / y$ and $\left(w_{5}, v\right) \in y$. By the definition of composition we get that $\left(u, w_{2}\right) \in x ; x \backslash x_{1}$ and $\left(w_{4}, v\right) \in y_{2} / y ; y$. Then by the definition of the residuals we have that $\left(u, w_{2}\right) \in x_{1}=x_{1}^{\prime}+x_{1}^{\prime \prime},\left(u, w_{3}\right) \in x_{2}=x_{2}^{\prime}+x_{2}^{\prime \prime},\left(w_{4}, v\right) \in y_{2}=y_{2}^{\prime}+y_{2}^{\prime \prime}$ and $\left(w_{3}, v\right) \in y_{1}=y_{1}^{\prime}+y_{1}^{\prime \prime}$.

For a contradiction assume that $(u, v) \notin \sigma_{2}$. Then $\left(w_{3}, v\right) \notin y_{1}^{\prime}$, otherwise we would have $(u, v) \in x ; x \backslash x_{1} ;\left(x ; x \backslash x_{1}\right) \backslash x_{2} ; y_{1}^{\prime}$. Hence $\left(w_{3}, v\right) \in y_{1}^{\prime \prime}$. Then $\left(u, w_{2}\right) \notin$ $x_{1}^{\prime}$, otherwise we get $(u, v) \in x_{1}^{\prime} ;\left(x ; x \backslash x_{1}\right) \backslash x_{2} ; y_{1}^{\prime \prime}$. Hence $\left(u, w_{2}\right) \in x_{1}^{\prime \prime}$. Then $\left(w_{4}, v\right) \notin y_{2}^{\prime}$, otherwise we get $(u, v) \in x_{1}^{\prime \prime} ;\left(x ; x \backslash x_{1}\right) \backslash x_{2} ; y_{1} /\left(y_{2} / y ; y\right) ; y_{2}^{\prime}$. Hence $\left(w_{4}, v\right) \in y_{2}^{\prime \prime}$. Then $\left(u, w_{3}\right) \notin x_{2}^{\prime}$, otherwise we get $(u, v) \in x_{2}^{\prime} ; y_{1} /\left(y_{2} / y ; y\right) ; y_{2}^{\prime \prime}$. But then $\left(u, w_{3}\right) \in x_{2}^{\prime \prime}$, whence $(u, v) \in x_{2}^{\prime \prime} ; y_{1} /\left(y_{2} / y ; y\right) ; y_{2} / y ; y$. That is, $(u, v) \in \sigma_{2}$ contrary to the assumption.

On the other hand, the evaluation $\epsilon$ given by

$$
\epsilon(x)=a \quad \epsilon\left(x_{i}^{\prime}\right)=a_{i}^{\prime} \quad \epsilon\left(x_{i}^{\prime \prime}\right)=a_{i}^{\prime \prime} \quad \epsilon(y)=b \quad \epsilon\left(y_{i}^{\prime}\right)=b_{i}^{\prime} \quad \epsilon\left(y_{i}^{\prime \prime}\right)=b_{i}^{\prime \prime}
$$


falsifies $e_{n}$ in $\mathfrak{A}_{n}$. Indeed, $a \backslash a_{i}$ and $b_{i} / b$ equal the identity $1^{\prime}$ for all $1 \leq i \leq n$ (since $a \leq a_{i}=a_{i}^{\prime}+a_{i}^{\prime \prime}$ and there is no nonzero element $z$ other than the identity such that $\left.a ; z \leq a_{i}\right)$. Since $z=z ; 1^{\prime}$, we get that every term between $x$ and $y$ in $\tau_{n}$ evaluates to $1^{\prime}$. Hence $\tau_{n}$ evaluates to $a ; b=1$, By the same reasoning we get that every element of the sum in $\sigma_{n}$ evaluates to $o$. Thus $\epsilon\left(\tau_{n}\right)=1 \not \leq o=\epsilon\left(\sigma_{n}\right)$.

To finish the proof, let $\varphi$ be an arbitrary first-order logic formula valid in $\mathrm{R}\left(+, ;,^{*}, /, \backslash, \smile, 0,1^{\prime}, 1\right)$. Then $\varphi$ is valid in $\mathfrak{A}$, because the latter is representable. Since $\mathfrak{A}$ is an ultraproduct of the $\mathfrak{A}_{n}$ 's, there is an $n$ such that $\varphi$ is valid in $\mathfrak{A}_{n}$. Since the equation $e_{n}$ is not valid in $\mathfrak{A}_{n}$, we have that $\varphi$ does not imply $e_{n}$, though the latter is an equation valid in $\mathrm{R}(+, ;, /, \backslash)$.

As a corollary we get the following.

Theorem 3.3. The equational theory of relational action algebras is not finitely axiomatizable over the equational theory of relational Kleene algebras, i.e., there is no finite set of $\mathrm{Eq}(\mathrm{RAA})$ which together with $\mathrm{Eq}(\mathrm{RKA})$ would imply $\mathrm{Eq}(\mathrm{RAA})$.

Proof. Assume the contrary, i.e., that there is a finite set $E$ which would axiomatize the equational theory $\mathrm{Eq}(\mathrm{RAA})$ of RAA over the equational theory $\mathrm{Eq}(\mathrm{RKA})$ of RKA. Since the finite set $E^{\prime}$ of equational axioms of AA in [13] implies $\mathrm{Eq}(\mathrm{RKA})$, then the finite set $E \cup E^{\prime}$ would axiomatize $\mathrm{Eq}(\mathrm{RAA})$, which is impossible according to Theorem 3.2.

\section{Action Lattices}

In this section we look at similarity types that include the meet operation as well. Since Theorem 3.2 does not apply to signatures that include meet, we need another construction to show nonfinite axiomatizability of the equational theory.

The following result follows from [7, Theorem 2.3 and Corollary 4.4.]. The construction in [7] is rather involved, since we needed dense algebras (where $x \leq$ $x ; x$ is valid) so that they can be applied to relevance logic. A simpler construction is available from [11] that has been used to show nonfinite axiomatizability of the equational theories of some residuated algebras in [12]. The Kleene star operation was not considered in [12], but it can be easily added to the signature. Also, complementation - can be included in the signature; in the representable algebras - is interpreted as complementation with respect to the largest element of the algebra.

Theorem 4.1. Let $\{\cdot,+, ;, \backslash\} \subseteq \Lambda \subseteq\left\{\cdot,+,-, ;,{ }^{*}, /, \backslash,{ }^{\smile}, 0,1^{\prime}, 1\right\}$. The equational theory of $\mathrm{R}(\Lambda)$ is not finitely axiomatizable.

Moreover, there is no strong, finite quasi-axiomatization of $\mathrm{R}(\Lambda)$. In fact, there is no first-order logic formula valid in $\mathrm{R}\left(\cdot,+,-, ;,{ }^{*}, /, \backslash,{ }^{\smile}, 0,1^{\prime}, 1\right)$ which implies all the equations valid in $\mathrm{R}(\cdot,+, ;, \backslash)$.

Proof. We recall the main features of the algebras $\mathfrak{A}_{n}=\left(A_{n},-, \cdot,+, ;,{ }^{\smile}, 0,1^{\prime}, 1\right)$ of [11]. $\mathfrak{A}_{n}$ has the following atoms (minimal, nonzero elements): identity $1^{\prime}, q_{i}$ 
for $1 \leq i \leq m$, and $p_{j}$ for $1 \leq j \leq n$ with $m=3 \cdot n$ !. Every atom is self converse: $x^{\smile}=x$. Composition is defined so that

$$
\begin{aligned}
q_{i+1} & \leq p_{1} ; q_{i} & & \text { for every } 1 \leq i<m \\
0 & =q_{r} \cdot q_{s} ; q_{t} & & \text { for every } 1 \leq r, s, t \leq m \\
0 & =p_{l} \cdot p_{l} ; p_{l} & & \text { for every } 1 \leq l \leq n .
\end{aligned}
$$

The elements of $\mathfrak{A}_{n}$ are the subsets of the atoms, and $+, \cdot,-$ are defined as the corresponding set theoretic operations. Composition ; and converse ${ }^{\sim}$ distribute over + . We define $x \backslash y:=-\left(x^{\smile} ;-y\right)$ and $x / y:=-\left(x ; y^{\smile}\right)$. It follows that (the $\{\cdot,+, ;\}$-reduct of) $\mathfrak{A}_{n}$ is not representable (since that would imply the existence of a colouring of the edges of a total graph of $m$ vertices with $n$ colours without monochromatic triangles; an impossible task). See Figure 2, where every dotted arrow should have a color $p_{i}$ for some $1 \leq i \leq n$, and $[1,12]$ for further details.

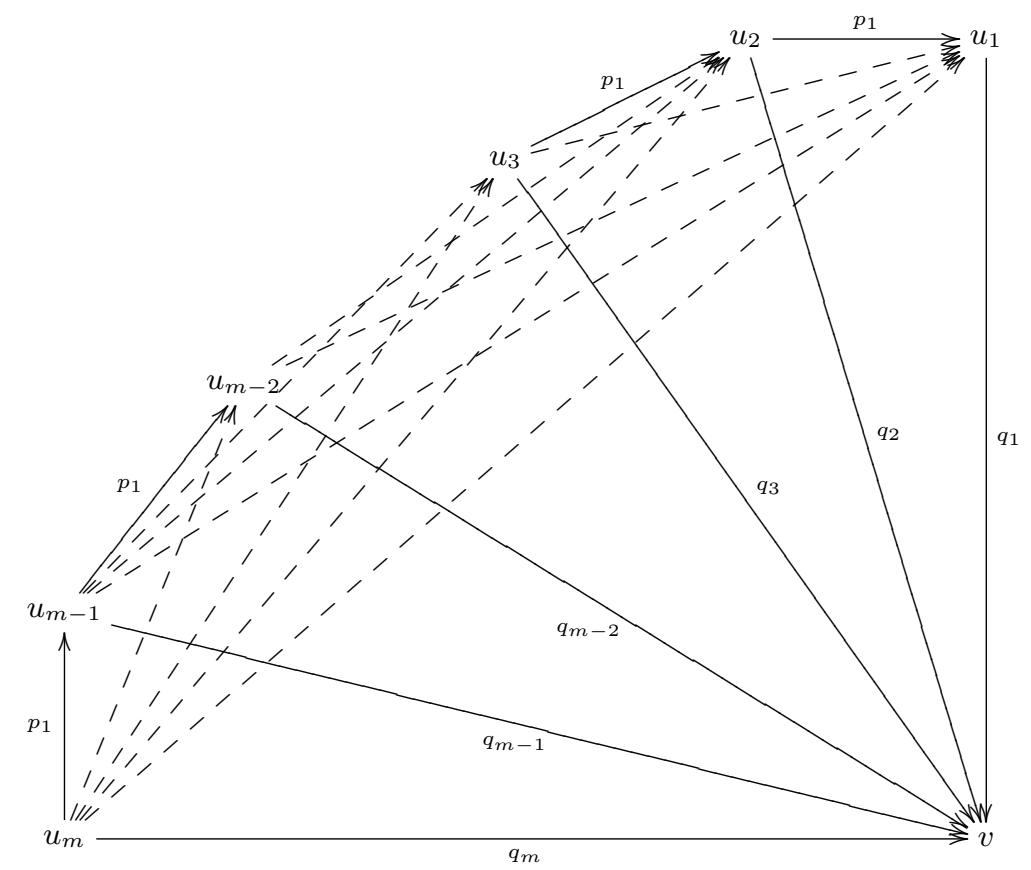

Fig. 2. The reason for non-representability

Furthermore, for every nonidentity atom $x$, we have that $x^{3}:=x ; x ; x=1$ in $\mathfrak{A}_{n}$. Thus we can define Kleene star in $\mathfrak{A}_{n}$ as

$$
x^{*}= \begin{cases}1^{\prime} & x \in\left\{0,1^{\prime}\right\} \\ 1 & \text { otherwise }\end{cases}
$$


It is shown in [11] that any nonprincipal ultraproduct $\mathfrak{A}$ of the $\mathfrak{A}_{n}$ 's is representable. By the above definition of Kleene star in $\mathfrak{A}_{n}$, it follows that we can define ${ }^{*}$ in the same way in $\mathfrak{A}$ and that the representation respects ${ }^{*}$ as well.

It is shown in [12] that there are equations $e_{n}$ in the language $\{\cdot,+, ;, \backslash\}$ such that they witness the nonrepresentability of $\mathfrak{A}_{n}$ :

- $e_{n}$ fails in the $\{\cdot,+, ;, \backslash\}$-reduct of $\mathfrak{A}_{n}$,

$-e_{n}$ is valid in representable algebras.

We give some intuition on the construction of $e_{n}$. To begin with, it is not difficult to define a quasi-equation $e_{n}^{\prime}$ expressing that, given the definition of composition in (1) and that the composition $p_{1}^{i}$ of $i$ many $p_{1}$ is below $\sum_{j} p_{j}+\sum_{k} q_{k}$, either (3) (there are no monochromatic $p$-triangles) or (2) (there are no $q$-triangles) is violated, or some of the $q$-atoms coincide. For instance, we can define $e_{n}^{\prime}$ as

$$
\begin{aligned}
& \bigwedge_{i} y_{i+1} \leq x_{1} ; y_{i} \wedge \bigwedge_{i} x_{1}^{i} \leq \sum_{l} x_{l}+\sum_{j} y_{j} \rightarrow \\
& y_{m} \leq \sum_{i, j, l} x_{1}^{i} ;\left(x_{l} \cdot x_{l} ; x_{l}\right) ; y_{j}+\sum_{i, j, k} x_{1}^{i} ;\left(y_{m-i+1} \cdot y_{j} ; y_{k}\right)+\sum_{i, j \neq k} x_{1}^{i} ;\left(y_{j} \cdot y_{k}\right)
\end{aligned}
$$

where $i, j, k$ range between 1 and $m$ and $l$ ranges between 1 and $n$. By the definition of composition, $e_{n}^{\prime}$ fails in $\mathfrak{A}_{n}$ (the antecedent is true, but every element of the sums in the consequent evaluates to 0 when we evaluate $x_{l}$ to $p_{l}$ and $y_{i}$ to $q_{i}$ ). On the other hand, in representable algebras, assuming the antecedent, every $y_{m}$-edge in the representation has a decomposition indicated by one of the elements in the sums (otherwise the representation would yield a graph colouring without monochromatic triangles). Finally, using the expressive power of the residuals, $e_{n}^{\prime}$ can be equivalently reformulated as an equation $e_{n}$ - for the technical details we refer the interested reader to [12].

Hence there are algebras, the $\Lambda$-reducts of $\mathfrak{A}_{n}$, which are not in the variety generated by $\mathrm{R}(\Lambda)$ but their nonprincipal ultraproducts are in this variety. From here on the proof ends as in that of Theorem 3.2.

Similarly to action algebras we get the following corollary.

Corollary 4.2. The equational theory of relational action lattices is not finitely axiomatizable over the equational theory of relational Kleene algebras, i.e., there is no finite set of $\mathrm{Eq}(\mathrm{RAL})$ which together with $\mathrm{Eq}(\mathrm{RKA})$ would imply $\mathrm{Eq}(\mathrm{RAL})$.

\section{Conclusion and Open Problems}

We mentioned in the introduction that KA provides a strong, finite quasi-axiomatization of RKA. That is, the equational theory of RKA follows from a finite conjunction $\varphi$ of quasi-equations valid in RKA; see Kozen [9] for this fact and [6] for references to related results. We proved that the analogous result does not hold for representable residuated algebras, even if we allow $\varphi$ to be any firstorder logic formula valid in representable algebras. Our first question is whether 
we can relax the requirement that $\varphi$ be valid in representable algebras; and if we relax this condition whether we can choose $\varphi$ to be a conjunction of quasiequations. This would mean to find a finitely axiomatized quasi-variety $\mathrm{Q}$ which generates the same variety as the representable algebras (but we do not require $\mathrm{RAA} \subseteq \mathrm{Q}$ or $\mathrm{RAL} \subseteq \mathrm{Q}$ ). For motivation we mention that such a quasi-variety would provide us with a finite quasi-equational axiomatization from which we could derive precisely those equations which are valid in representable algebras. Thus, in this respect, validity of the axioms that are not equations is not crucial.

Problem 5.1. Do RAA and RAL have finite quasi-axiomatizations? That is, are there finitely axiomatized quasi-varieties $Q_{1}$ and $Q_{2}$ such that they generate the same varieties as generated by RAA and RAL, respectively?

By Kozen's result in [9] and our Theorem 3.1 we get, with an argument analogous to the proof of Theorem 3.3, that the quasi-equational theory of RKA is not finitely axiomatizable over the equational theory of RKA. In view of our Theorem 3.2, the same argument does not work for RAA, i.e., it is possible perhaps that the quasi-equational theory of RAA is finitely axiomatizable over the equational theory of RAA. We can hope even for the two theories to coincide in this case, because of the following. As Pratt's work shows, the presence of the residuals of composition allows us to express certain quasi-equations as equations. We are not aware of any general result that would characterize precisely when this can be done. Perhaps this can be done for sufficiently many quasi-equations. Hence we formulate the following question.

Problem 5.2. Do the quasi-variety and the variety generated by RAA (and by RAL) coincide?

Finally, we mention the same finite axiomatizability problem we addressed in this paper in the context of language algebras. It is well known that the equational theories of relational and language Kleene algebras coincide. However, their quasi-equational theories already differ, there are fewer language Kleene algebras than relational ones. Also the equational theories differ if we include meet into the similarity type; see [3] on the connections between the relational and the language models of Kleene algebras/lattices.

Problem 5.3. Are the equational theories of action algebras and action lattices interpreted over languages finitely axiomatizable? If the answer is negative, do they have (strong) finite quasi-axiomatizations?

In connection with this problem we mention that the algebras we used to prove nonfinite axiomatizability in the relational case are of limited use, since their ultraproduct is not representable as a language algebra. The reason is that it contains elements $x$ that are disjoint from the identity $1^{\prime}$, yet $x ; x \geq 1^{\prime}$. This cannot happen in a language algebra, since $1^{\prime}$ is represented as the singleton language containing only the empty word. 


\section{References}

1. H. Andréka. Representation of distributive lattice-ordered semigroups with binary relations. Algebra Universalis, 28:12-25, 1991.

2. H. Andréka and Sz. Mikulás. Axiomatizability of positive algebras of binary relations. Algebra Universalis, 66(1):7-34, 2011.

3. H. Andréka, Sz. Mikulás, and I. Németi. The equational theory of Kleene lattices. Theoretical Computer Science, 412:7099-7108, 2011.

4. J.H. Conway. Regular Algebra and Finite Machines. Chapman and Hall, 1971.

5. S. Crvenković, I. Dolinka, and Z. Ésik. The variety of Kleene algebras with conversion is not finitely based. Theoretical Computer Science, 230:235-245, 2000.

6. Z. Ésik and L. Bernátsky. Equational properties of Kleene algebras of relations with conversion. Theoretical Computer Science, 137:237-251, 1995.

7. R. Hirsch and Sz. Mikulás. Positive reducts of relevance logic and algebras of binary relations. Review of Symbolic Logic, 4(1):81-105, 2011.

8. P. Jipsen. From semirings to residuated Kleene lattices. Studia Logica, 76(2):291303, 2004

9. D. Kozen. A completeness theorem for Kleene algebras and the algebra of regular events. Information and Computation, 110:366-390, 1994.

10. D. Kozen. On action algebras. In J. van Eijck and A. Visser, editors, Logic and Information Flow, pages 78-88. MIT Press, 1994.

11. R.D. Maddux. Non-finite-axiomatizability results for cylindric and relation algebras. Journal of Symbolic Logic, 54(3):951-974, 1989.

12. Sz. Mikulás. On representable ordered residuated semigroups. Logic Journal of the IGPL, 19(1):233-240, 2011.

13. V. Pratt. Action logic and pure induction. In J. van Eijck, editor, Logics in AI: European Workshop JELIA '90, pages 97-120. Springer, 1990.

14. V.N. Redko. On defining relations for the algebra of regular events. Ukrain. Mat. Z., 16:120-126, 1964. In Russian. 